\title{
A pie: wandering y cotidianidad en Simone de Eduardo Lalo
}

\author{
María Paz Oliver
}

Published online: 18 February 2015

(C) The Author(s) 2015. This article is published with open access at Springerlink.com

\begin{abstract}
This article explores the relationship between wandering and everyday life in Simone (2012), a novel by the Puerto Rican writer Eduardo Lalo. Wandering, defined as an aimless movement around the city, is not only an instance to reflect and discover by walking the everyday life, but also to express a critical perspective towards the structures of power that articulates the daily life of San Juan. If the everyday relates to all daily activities, wandering is a representative movement of the rhythm of the city, which also enables to reveal the subversive potential of the everyday. Considering the figure of the flâneur, this article will analyze the way in which wandering is an instance to perceive the elusive and fragmentary nature of the everyday, which traces an emotional cartography of the postmodern city. Moreover, in the context of globalization, this aimless strolling has the potential to reflect on the non-places of everyday life. Finally, from the perspective of psychogeography, the article proposes that wandering in Lalo's novel provides a critical view on the ideological dimension of everyday life, and particularly on its monotony and alienating effect.
\end{abstract}

Keywords Wandering - Everyday life · Eduardo Lalo · Flâneur .

Psychogeography

Tal como observa Juan Duchesne Winter (2011, 32-33), la literatura puertorriqueña del siglo XXI tiende a cuestionar el régimen colonial norteamericano que rige hasta el día de hoy. Si bien durante el siglo XX buena parte de la literatura de Puerto Rico se inscribió como un producto de ese dominio político y cultural, en el último tiempo la búsqueda de una identidad propia alejada tanto de la cultura estadounidense como de los estereotipos caribeños, ha conformado un panorama literario

M. P. Oliver $(\square)$

Radboud Universiteit Nijmegen, Erasmusgebouw, Erasmusplein 1, 6525 HT Nijmegen,

The Netherlands

e-mail: mariapazoliver@gmail.com 
especialmente atractivo para comprender el lugar de Puerto Rico como cultura de resistencia.

Galardonado con el Premio Rómulo Gallegos en 2013 por su novela Simone (2012), Eduardo Lalo es una de las voces más reconocidas de la literatura latinoamericana actual, y particularmente de una nueva generación de escritores que cuestiona las formas de representación y la marginalidad cultural del Caribe desde una perspectiva crítica y postmoderna. A medio camino entre el ensayo, la poesía y la ficción, la obra de Eduardo Lalo aborda la situación actual de Puerto Rico no sólo como la frontera territorial y cultural de América Latina, que ambivalentemente se debate entre la preservación de la lengua castellana y el dominio de la metrópoli, sino también como la condición de ser, como él mismo observa, un "país invisible" (Cella 2013).

Al igual que en otras obras, ${ }^{1}$ en Simone Eduardo Lalo traslada la pregunta por la identidad puertorriqueña a la cotidianidad de la vida urbana de San Juan y a la experiencia psicogeográfica de la ciudad. ${ }^{2}$ De manera análoga al proyecto de Perec en Tentative d'épuisement d'un lieu parisien (1975), en las caminatas y en los vagabundeos sin rumbo por la ciudad, Lalo redirige la mirada hacia los ritmos de la vida diaria, para así describir y revelar la experiencia de una cotidianidad que, aunque se define por lo que pasa cuando no pasa nada, tal como anota Perec en su registro de las calles parisinas, rescata el valor creativo del wandering para explorar su dimensión espacial y urbana. De este modo, se trata de ver cómo las improvisadas caminatas a lo largo de la ciudad se convierten en un método de observación y análisis de los diversos nombres, objetos y situaciones de la vida cotidiana que, al modo de un inventario que continuamente se renueva en esos movimientos, intenta resignificar y develar el potencial crítico del espacio cotidiano.

\section{El wandering cotidiano}

Si hay un punto en común en los estudios sobre lo cotidiano es la dificultad para definir este concepto. Ya sea por su incuestionable evidencia o porque remite a lo que acontece en un presente inmediato, la idea de lo cotidiano se aplica a todo aquello que sucede de manera habitual y que, por su mismo carácter rutinario y elusivo, suele quedar excluido de todo registro (Highmore 2002; Felski 1999-2000). En lo cotidiano, de algún modo, se cifraría la tensión entre la indeterminación de las actividades asociadas a una rutina y la falta de una distancia reflexiva al momento de convertirlas en objeto de estudio. Como observa Maurice Blanchot, lo cotidiano se sitúa en un presente que está constantemente sucediendo, pero que a la vez no

\footnotetext{
${ }^{1}$ Pensamos particularmente en En el Burger King de la calle San Juan (1986), Ciudades e islas (1995) y donde (2005).

${ }^{2}$ El jurado de la XVIII edición del Premio Internacional Novela Rómulo Gallegos, compuesto por Ricardo Piglia, Juan R. Duchesne Winter y Luis Duno-Gottberg, destacó que en la novela de Lalo: "Las modalidades de la crónica, el diario, la psicogeografía urbana, el collage de citas, el aforismo y arte conceptual convergen en una aventura magistralmente contada donde se apuesta, pese al culto al fracaso que embarga al narrador, a la capacidad reivindicadora de la literatura, el amor y el ensueño en el mundo desencantado del capitalismo tardío” (2013).
} 
termina nunca de realizarse, es decir, remite a un movimiento en el que paradójicamente percibimos que no pasa nada: “il est toujours déjà là, mais qu'il soit là ne vérifie pas son accomplissement: au contraire, toujours inaccompli dans sa réalisation même qu'aucun événement, si important, si insignifiant qu'il soit, ne pourra produire. Rien ne se passe, voilà le quotidien" (Blanchot 1969, 360). En esta misma línea, y por oposición a lo extraordinario—o a aquellos acontecimientos que conforman propiamente el objeto de la prensa-, para Perec lo cotidiano refiere a aquello a lo que estamos tan acostumbrados que no somos capaces de percibirlo, esto es, a lo que denomina lo 'infraordinario'. ${ }^{3}$ Así, la indeterminación de lo cotidiano diluye la posibilidad de delimitar de manera clara qué actividades comprende y cómo éstas se describen. La propuesta de Perec apunta, entonces, a redescubrir lo cotidiano a través de ejercicios que se sirven de la observación y la clasificación para interrogar las experiencias del día a día y devolverle, en definitiva, el sentido a una cotidianidad que sería el reflejo de lo que somos: "Quizás se trate finalmente de fundar nuestra propia antropología: la que hablará de nosotros, la que buscará en nosotros lo que durante tanto tiempo hemos copiado de los demás. Ya no lo exótico, sino lo endótico" (Perec 1973, 23).

Simone es una novela que se sitúa tentativamente entre el registro etnográfico de un escritor y profesor universitario — con una evidente carga autoficcional—que lleva un diario sobre sus andanzas por San Juan y la historia sobre la persecución y el encuentro amoroso con una desconocida que le envía mensajes firmados indistintamente con el seudónimo Simone, Simone Weil y S.W. Los apuntes sobre las caminatas por los distintos barrios de San Juan funcionan como un ensayo sobre la percepción de lo cotidiano, en un intento por devolverle el asombro a la mirada ante los espacios y objetos que componen la vida diaria. Así, el punto de partida para las observaciones del protagonista es la inestabilidad y la dispersión de un presente determinante a la hora de definir sus reflexiones a lo largo de los paseos por los barrios y centros comerciales de San Juan:

Escribir fragmentos, escribir notas en una libreta al vuelo de los días, es lo que más se acerca a una escritura que no sabe que miente. Luego, cuando se reelabora, se crean los subterfugios y establecen las maneras de no decir o de no decir del todo [...] No sé lo que pasará mañana. No sé lo que escribiré después. Tengo toda la escritura por delante $(2012,58)$.

$\mathrm{Al}$ igual que un diario íntimo, las anotaciones plantean la tensión entre el aspecto elusivo del presente y el deseo de ordenar los pensamientos en torno a la rutina. Así como el diario íntimo utiliza una escritura cotidiana particularmente abierta para expresar la inestabilidad y fragmentariedad del discurso del yo (Blanchot 1959, 207), aquellas notas subrayan la fragilidad e inmediatez de lo cotidiano a través de un relato que tiende a descentrarse o irse del tema para así destacar la contingencia de ese ritmo. Esta preferencia por la escritura fragmentaria entra, además, en sintonía con los intempestivos mensajes que recibe el protagonista, con el posible

\footnotetext{
3 Perec postula este neologismo en el ensayo “Approches de quoi?” (Perec 1973) para destacar el carácter habitual de lo cotidiano y cómo se opone a lo que comúnmente se entiende por extraordinario. Ben Highmore destaca que Perec utiliza el término 'infraordinario' para describir una cotidianidad "that is neither ordinary nor extraordinary, neither banal nor exotic" (Highmore 2002, 176).
} 
sentido oculto de esa serie de oraciones inconexas, y particularmente con una de ellas, en referencia a Walter Benjamin, que como un reflejo de la propia obra subraya ese potencial intertextual del fragmento: "en nuestro tiempo la única obra realmente dotada de sentido-de sentido crítico también-debería de ser un collage de citas, fragmentos, ecos de otras obras" $(2012,53)$.

La casualidad e inestabilidad de lo cotidiano se expresa, por lo tanto, de manera análoga en el acto mismo de caminar por la ciudad y transferir esa experiencia en la escritura. En Simone la espontaneidad propia de la caminata predispone para una actividad meditativa que conecta las transformaciones del paisaje con el fluir de los pensamientos del protagonista, en un proceso donde la mirada sobre lo cotidiano se configura desde la fragilidad propia del presente: "Pensar desde la nada, desde este nada pasa, desde aquí. Y lo digo con la euforia del que ha perdido la esperanza y sigue y pervive" $(2012,19)$. Acorde a la percepción de que "no pasa nada", el aspecto fragmentario de la escritura intenta describir la naturaleza elusiva de lo cotidiano a través de una narrativa que, al igual que los desplazamientos del protagonista por las calles de la ciudad, digresivamente se renueva en los cambios de tema de cada entrada del cuaderno:

Es tremendo lo que pasa sin que nada pase. Aquí estoy, sentado ante un café, leyendo un libro, escribiendo en un cuaderno.

'La humanidad publica un libro cada medio minuto', Gabriel Zaid, Los demasiados libros.

Diego, que desde que trabaja en la banca, vive temporadas cada vez más largas en el extranjero, me va relegando como tantos otros elementos de su pasado (2012, 33).

Apelando al carácter residual de lo cotidiano, Lalo trabaja una escritura también del detalle y de lo insignificante que, siguiendo las ideas de Henri Lefebvre, concibe lo cotidiano como el sustrato de toda actividad especializada. Lo que queda, aquel "ce qui reste" en palabras de Lefebvre (1947, 97), a pesar de estar relacionado con las acciones que acontecen diariamente, siempre queda fuera de todo análisis y, en consecuencia, se opone directamente a lo extraordinario, a lo artístico e, incluso, a la actividad reflexiva (Lefebvre 1947, 97-98). De ahí que la actitud para contemplar lo cotidiano necesite de un tipo de conocimiento especialmente práctico y transitorio que sea capaz de captar esa misma futilidad que rodea el día a día. Como sostiene William Hazlitt en el ensayo "On Going a Journey” (1822), la caminata favorece un método de conocimiento sintético por sobre uno analítico, es decir, a lo largo de un paseo se captan de manera aleatoria las ideas para luego, eventualmente, ser analizadas al término de ese viaje: "I am content to lay in a stock of ideas then, and to examine and anatomise them afterwards. I want to see my vague notions float like the down of the thistle before the breeze, and not to have them entangled in the briars and thorns of controversy" (Hazlitt 1822, 183). A diferencia de un acto reflexivo consciente, el efecto de distracción propio de la caminata libre por la ciudad promueve un conocimiento intuitivo frente al escurridizo devenir de lo cotidiano. Por eso, como observa Michael Sheringham, la aprehensión de lo cotidiano exige un acto de reconocimiento de las cosas que componen la vida diaria, 
esto es, una transformación de nuestra atención frente a lo ordinario, de tal modo que mediante una inversión de la mirada aquella inestabilidad de lo cotidiano se comprenda como un acto performativo y no como una simple constatación de ciertos hechos u objetos (Sheringham 2000, 187-188).

La representación de lo cotidiano supone, por lo tanto, la puesta en escena de una mirada que se reconfigura en el encuentro con la fugacidad de los espacios y objetos de la cotidianidad y, particularmente en el caso de Lalo, en el acto de desplazarse por la ciudad. Es decir, el caminante y lo cotidiano se definen y modifican recíprocamente a lo largo del desplazamiento, pues así como el entorno espacial influye en la percepción de lo cotidiano-desde la dispersión y contingencia de un presente que continuamente se renueva-, el acto mismo de observar y describir esos espacios les añade una carga emotiva que los personaliza. Así, a través del movimiento, las caminatas de este escritor van trazando una línea obsesiva que se mueve entre la búsqueda literaria y personal, y que a mayor escala termina por convertirse en una cartografía urbana que le devuelve el sentido emotivo a los espacios cotidianos.

¿Qué son estas calles sino la vida mía? El tiempo circulando como agua o viento, un cuerpo que se irá haciendo pequeño y frágil junto a las cunetas que van siempre en la misma dirección, por el camino que también es mío. Las ciudades le importan más a los que van en la misma dirección a sus cunetas, a los que caminan a su misma altura. A ningún dueño de la ciudad, a ninguno de sus alcaldes, le importa la ciudad como a mí me ha importado, porque yo sé que no tengo salida, que nunca me podré ir de aquí $(2012,65)$.

\section{Los 'no lugares' de la cotidianidad}

En el contexto de una globalización que interfiere directamente en la delimitación y percepción de los espacios públicos, la caminata sin destino por las calles propone la pregunta por la identidad de los espacios cotidianos asociados a la vida urbana. De esta manera, la dimensión global de la ciudad contemporánea replantea la configuración de los espacios y su valor simbólico desde una perspectiva transnacional (Sassen 2007, 102) que afecta decisivamente la experiencia cotidiana de esos lugares, sobre todo si se considera el caso en particular de Puerto Rico respecto al dominio político y cultural estadounidense. Este debilitamiento en la identidad de los lugares proyecta una progresiva homogeneización y falta de autenticidad del paisaje que incluso termina por destruir el sentido mismo del lugar, como sucede, por ejemplo, en las autopistas de alta velocidad, como observa Edward Relph, que se desconectan de la geografía que las rodea (Relph 1976, 90-92). La escritura de los apuntes de Lalo da cuenta de un desplazamiento que tiende a cuestionar el supuesto anonimato de los llamados 'no lugares' de la modernidad, propuestos por Marc Augé en Non-lieux. Introduction à une anthropologie de la surmodernité (1992), que aluden a los lugares de tránsito y consumo del mundo contemporáneo: los centros comerciales, los aeropuertos, las estaciones, los medios de transporte, los supermercados, entre otros. 
Sin dejar de aparecer como lugares de tránsito representativos de la sociedad de consumo, ${ }^{4}$ en Simone los 'no lugares' descritos a lo largo de las caminatas-los centros comerciales y el aeropuerto de San Juan, específicamente-más bien entran en tensión con su aparente anonimato para eventualmente recobrar un sentido a través del desplazamiento urbano. Para Lalo la caminata contiene un potencial renovador frente a la ambigüedad e indeterminación del espacio cotidiano, de modo que en ese acto lo que en principio se concibe como un lugar indiferenciado y carente de identidad, se transforma en lo que Yi-Tu Tuan entiende propiamente por lugar, esto es, aquel espacio que pasa a ser conocido y dotado de valor mediante las prácticas cotidianas de los habitantes (Tuan 1977, 6). Por ejemplo, en la historia que le había contado su amigo Diego, sobre un jubilado que había vivido en Nueva York y que cada semana pasa una noche en el aeropuerto de San Juan para luego devolverse a la mañana siguiente sin haber tomando ningún avión (2012, 25-26), Lalo sugiere una forma de reapropiación de aquel lugar en el absurdo de esa situación. Desde el momento en que el aeropuerto pierde su condición de lugar de tránsito en relación a un viaje, cambia en el espectador la experiencia de ocupar aquel lugar y, como diría Perec, se invierte la mirada respecto a la cotidianidad de ese espacio. Ese efecto de extrañamiento le devuelve a este 'no lugar' un sentido de pertenencia y una emotividad a través de la experiencia íntima de recorrer esos espacios, evocando el recuerdo de un viaje que le restituye la identidad al aeropuerto. Como un modo de recuperar el sentido de una vivencia genuina, con aquella visita el aeropuerto se transforma también en un símbolo de las ruinas desde donde se proyecta la memoria personal.

Así también las caminatas por el centro comercial Santa Rosa en Bayamón plantean una tensión entre el carácter anónimo y masivo de ese lugar y su potencial estético. Contradiciendo la ética del consumo, la caminata por el sólo gusto de descubrir esos espacios es una actividad que se opone a la lógica de ese 'no lugar', y que en ese acto de apropiación lo transforma en un material para la ficción. De ahí que las referencias a estos espacios también manifiesten una mirada desencantada frente a las políticas globalizadas que dominan la cotidianidad del entorno urbano: "La rutina de la ciudad: la soledad transita por autopistas y recala en gasolineras que abren veinticuatro horas" $(2012,40)$. Desde la privacidad de la escritura, Lalo cuestiona la condición de 'no lugar' del espacio cotidiano y expresa una mirada ambivalente que se mueve entre la crítica distante y un cierto atractivo nostálgico:

Mi imagen de un centro comercial: un hombre solo, sentado ante una mesa, en la terraza repleta de restaurantes de comida basura, con un café y una libreta. A mis pies, una mochila con libros, otro cuaderno y dos plumas fuentes. Llevo horas aquí y no he comprado nada, ni siquiera un libro. Extraño, extrañísimo

\footnotetext{
${ }^{4}$ Es indudable la resonancia de San Juan como escenario representativo además de la sociedad capitalista globalizada y dominada por la cultura estadounidense, como advierte el propio Lalo en su discurso al recibir el Premio Rómulo Gallegos: "Soy de ese lugar que acaso vivió la globalización antes que cualquier otra sociedad, aún antes de que existiera el término y el conocimiento, tanto de sus consecuencias como también de las formas de oponerla. Soy de un país que resistió solo, por la fuerza de su propia cultura, a las imposiciones imperiales del país que domina y seduce desde el comienzo del siglo XX” (Lalo 2013).
} 
ante todo lo que me rodea, pero para mí no existe en el mundo una imagen más hechizante y perturbadora $(2012,28)$.

Por otra parte, el wandering va creando un tejido urbano y un mapa simbólico de la ciudad a partir del cruce entre el desplazamiento casual y la experiencia del caminante frente a los lugares y 'no lugares' que integran la ciudad. Ese movimiento remite a un intento también de definir en ese recorrido su posible identidad, tal como sostiene Michel de Certeau: “Andar es no tener un lugar. Se trata del proceso indefinido de estar ausente y en pos de algo propio. El vagabundeo que multiplica y reúne la ciudad hace de ella una inmensa experiencia social de la privación de lugar" (de Certeau 1980, 116). Como acto performativo, la caminata renueva aquellos lugares carentes de identidad, reinterpretando transitoriamente el sentido de los espacios cotidianos. Así, como anota Lalo, mientras observa las vitrinas de las tiendas, el paisaje divisado a lo largo de la caminata configura: "una cartografía de las palabras de tantos seres anónimos, puestas en rectángulos de plástico o convertidas en estructuras de neón. [...] Son oraciones en la novela de la ciudad" $(2012$, 46). Frente al aparente anonimato de los espacios cotidianos, las descripciones y referencias concretas a esos espacios, y particularmente a los nombres de las calles, trazan un registro personal de la ciudad, un itinerario imaginario de lugares redescubiertos a través de la caminata, como se lee en las anotaciones sobre Río Piedras: "Pareciera, a pesar del caos, que todo estuviera en su sitio: la cantidad de hombres sin piernas en sus sillas de ruedas, los anuncios de Miss Millenium Model, la decoración neopsicodélica de la Cafetería Los Amigos, la fila de timbales en la vidriera de Casa Isern, el reloj de la estación del Tren Urbano" (2012, 64). A lo largo de las múltiples direcciones que toma la caminata, la acumulación de nombres propios llega a conformar una segunda geografía, imaginaria y personal, que se contrapone a la original. Como apunta de Certeau, los nombres propios organizan un discurso topográfico sobre la ciudad que, así como la vuelven habitable y reconocible, le imponen la identidad de quien transita esos lugares (de Certeau 1980, 118).

\section{Psicogeografía del espacio cotidiano}

En la línea de los experimentos situacionistas, las andanzas del protagonista por las calles de San Juan no son una estrategia para salir de la cotidianidad o quebrar la rutina, sino una actividad para precisamente descubrirla y extender la dimensión privada de lo cotidiano a la amplitud espacial de la calle, a sus ritmos y rutinas. En la caminata confluye, de este modo, la aprehensión de lo cotidiano y la delimitación de una geografía a escala humana que describe un espíritu transformador de la vida urbana. Emparentada con la llamada psicogeografía, proclamada por Guy Debord en los 50 para referir al estudio de la geografía y sus huellas sobre las emociones y el comportamiento humano en general (Debord 1955, 24), en la caminata se cruza la dimensión geográfica de la ciudad con su impacto psicológico, de tal manera que el vagabundeo urbano también se proyecta como una disposición para indagar en las lógicas de la vida cotidiana, y particularmente en su monotonía y efectos alienantes. 
En este sentido, la caminata posibilitaría una toma de conciencia de las lógicas de poder de la cotidianidad, como advierten los situacionistas, encaminada a desentrañar y subvertir los modos en que ésta controla y manipula cada espacio de la vida humana (Plant 1992, 58). La creación de situaciones, entre ellas, el wandering urbano, potenciaría una mirada crítica hacia las políticas de la sociedad de consumo que dominan el espacio cotidiano y que comúnmente pasan desapercibidas. Entre ellas, la dérive, definida por Jacques Fillon en "New Games!" (1954) como una "technique of locomotion without goal"5 (Fillon 1954, 155), reinscribe lúdicamente la tradición del flâneur ${ }^{6}$ en las calles de la ciudad contemporánea a través de movimientos guiados simplemente por la atracción o resonancia emotiva del espacio sobre el caminante. En la escritura de Lalo, el ejercicio de caminar a ciegas por la ciudad dibuja en ese espacio los vaivenes de la cotidianidad y la búsqueda de una perspectiva crítica sobre las condiciones en que ésta despliega sus mecanismos de poder. Simbólicamente, aquellas interminables caminatas reproducen por medio de la dérive las tensiones respecto a la búsqueda y el accidentado romance del escritor con Li Chao, el verdadero nombre de Simone, la muchacha china-puertorriqueña que a lo largo de la novela le envía mensajes con citas de libros de arte y literatura. El carácter errático de la caminata expresa, de este modo, el estado de ánimo del escritor y el deseo de hacer comunicable esa experiencia:

Transcurrieron días aciagos, en los que, como antídoto ineficaz a su ausencia, recorrí la ciudad sin orden ni destino. En las noches me sentaba a escribir la historia de nuestro encuentro como antes ella hacía sus dibujos: era el relato de una línea obsesiva que desembocaba en una geometría ilegible. [...] Este deambular por las calles y avenidas, sin ningún lugar a dónde ir, con la vaga esperanza de que algún día encontraría una salida que permitiera la ilusión momentánea de que se había partido, de que era posible otra vida o una situación que pareciera de verdad otro mundo $(2012,128)$.

Como también subraya de Certeau, la calle es el espacio por excelencia donde se expresan las estructuras de poder de la sociedad. La caminata, por lo tanto, sería un proceso donde el peatón se apropia de un sistema topográfico y define espacialmente el lugar, para a través de ese movimiento convertir el acto de caminar en un espacio de enunciación (de Certeau 1980, 141-142); en una retórica o en un estilo que

\footnotetext{
5 Merlin Coverley en su estudio sobe la psicogeografía (2010), refiere a la definición de dérive publicada en Internationale Situationniste \#1: "A mode of experimental behaviour linked to the conditions of urban society: a technique of transient passage through varied ambiances. Also used to designate a specific period of continuous deriving" (Coverley 2010, 93).

6 Charles Baudelaire en el ensayo "Le peintre de la vie moderne" (1863) postula una primera descripción del flâneur, luego retomada por Walter Benjamin en Das Passagen-Werk (1982), como una figura representativa del movimiento en las calles de la ciudad moderna, particularmente del París del siglo XIX, que se mueve ambivalentemente entre la atracción y la resistencia frente a la cultura del consumo. Zygmunt Bauman apunta que desde una perspectiva postmoderna, el flâneur sería tan sólo un símbolo de las actitudes de consumo del mundo contemporáneo: "The urge to 'wander without aim while shopping once in a while to look around' [...] has been caught upon mass industry [...] the freedom of the flâneur to set, playfully, the aims and meanings of his nomadic adventures, the original attraction of his lonely escapades, has been expropriated" (Bauman 1994, 154).
} 
intenta, a fin de cuentas, descifrar los misterios de la cotidianidad. La errancia en los movimientos sería un síntoma de esa tensión entre el poder de resistencia y dominación de lo cotidiano y la capacidad inventiva de la caminata, como una forma de desafiar el sentido ideológico de la vida diaria. Esta capacidad subversiva y creativa del wandering entra en disputa con el desarrollo de una ciudad contemporánea que progresivamente le da la espalda al caminante y que, como sostiene Merlin Coverley, predispone arbitrariamente un modo de recorrerla y de definir sus espacios: "Walking is seen as contrary to the spirit of the modern city with its promotion of swift circulation and the street-level gaze that walking requires allows one to challenge the official representation of the city by cutting across established routes and exploring those marginal and forgotten areas often overlooked by the city's inhabitants" (Coverley 2010, 12).

Frente a los mecanismos de poder contenidos en la cotidianidad, la caminata es una actividad desestabilizadora que, tal como apunta de Certeau, cuestiona el sistema codificado de la ciudad. A diferencia de las estrategias, que refieren a los cálculos conscientes del sujeto dentro de un sistema regulado, la caminata urbana actúa como una táctica de apropiación del espacio, esto es, como una acción improvisada que se vale de las circunstancias y de la oportunidad para organizar los movimientos dentro de la ciudad (de Certeau 1980, 40-45). De este modo, el wandering es un acto de apropiación subjetiva que busca revelar desde el presente cotidiano las lógicas de la ciudad como un gran sistema de poder. Si la ciudad metafóricamente funciona como un conjunto de reglas similar a una lengua, la caminata describe una forma de enunciación que críticamente quiebra ese orden y propone otra forma de cartografiar la ciudad. Sin embargo, como remarca Rebecca Solnit, el ilimitado crecimiento urbano de la ciudad contemporánea tiende a quitarle espacios al caminante y, por tanto, pone en riesgo esa capacidad expresiva de la caminata para describir una cartografía emotiva del movimiento urbano: "if a city is a language spoken by walkers, then a postpedestrian city not only has fallen silent but risks becoming a dead language whose colloquial phrases, jokes, and curses will vanish, even if its formal grammar survives" (Solnit 2001, 213). ${ }^{7}$

En la novela, la caminata dispone la mirada del protagonista para continuamente leer el paisaje urbano como un conjunto de signos dominado por la cultura globalizada que, al igual que los mensajes anónimos, reclama ser descifrado e interpretado críticamente: "Esto es lo que escucho y anoto en la calle. Tras las palabras queda el enigma. Pero todo sabe a plástico, a sol, a baterías doble A de un aparato hecho en China" $(2012,49)$. Desde esta perspectiva, en la caminata se entrecruza el paisaje de la ciudad con las imágenes subjetivas del caminante, creando una ficción fragmentaria y dispersa, que refiere al imaginario crítico de la ciudad contemporánea. A esta imagen además se le superponen los mensajes de Simone, para así recalcar de manera más explícita el espíritu subversivo de la caminata en la construcción del paisaje, como se lee en el primer mensaje escrito

\footnotetext{
7 Solnit refiere además a la teoría de Jean Christophe Bailly para remarcar cómo el imaginario social de la ciudad contemporánea, y particularmente el de París, se ve amenazado por la tiranía de una arquitectura que cada vez le deja menos espacio al caminante para desarrollar una actividad capaz de generar una gramática de la ciudad (Solnit 2001, 213).
} 
con tiza en el pavimento a la salida de la universidad: “¿Hasta qué punto podemos construir una sociedad basada en la mentira y el olvido?" (2012, 35).

Así como para Debord la supuesta trivialidad de lo cotidiano es una condición impuesta por la sociedad de consumo (Debord 1961, 240), la novela de Lalo explora el potencial subversivo de lo cotidiano para crear una imagen de San Juan fragmentaria y que, pese a su predominio, se resiste a la cultura globalizada. Ese carácter elusivo de lo cotidiano crea la tensión con el poder creativo de la caminata, pero a la vez se transfiere al acto mismo de caminar pues, como un flâneur nostálgico frente a las ruinas de la ciudad, el protagonista se confunde y pierde en las caminatas. Caminar en la novela de Lalo es un gesto que, así como abre la mirada crítica para descubrir la cotidianidad, difumina la identidad del protagonista en el anonimato de las calles. Como también observa Francisco Javier Avilés en Ciudades e islas, para Lalo la ciudad puertorriqueña sería un espacio ideal para perderse en su propia falta de identidad: "Lo póstumo se asume como manera de desaparición, una instancia en la conciencia del habitante de la ciudad en el que se reconoce que vivir las calles de la ciudad es aspirar a no ser visto: el anonimato es la metafísica de la ciudad" (Avilés 2012, 879). Pero, a diferencia del flâneur clásico, la caminata en la novela es también un ejercicio de resistencia y subversión frente a las políticas geográficas y culturales de San Juan. Si el flâneur es una figura típicamente moderna, que admira con curiosidad la ciudad capitalista, el caminante de Lalo es un antimoderno, pues reescribiendo la ciudad cuestiona las políticas desarrollistas, ${ }^{8}$ precisamente en un país marcado por el colonialismo estadounidense. De este modo, el wandering en la novela de Lalo es la actividad propia del outsider, de aquel que mediante aquellos trayectos sin rumbo se rebela frente a las dinámicas de progreso y consumo propias de la cotidianidad y de la geografía urbana; para proponer, en cambio, una cartografía crítica, imaginaria y emotiva de la ciudad contemporánea.

Open Access This article is distributed under the terms of the Creative Commons Attribution License which permits any use, distribution, and reproduction in any medium, provided the original author(s) and the source are credited.

\section{References}

Augé, M. (1992). Non-lieux. Introduction à une anthropologie de la surmodernité. Paris: Seuil.

Avilés, F. J. (2012). Estética del derrumbe: escritura y deambular urbano en la obra de Eduardo Lalo. Revista Iberoamericana, 241, 873-892.

Baudelaire, C. (1863). La peintre de la vie moderne. Paris: Éditions du Sandre. 2009.

Bauman, Z. (1994). Desert Spectacular. In K. Tester (Ed.), The flâneur (pp. 138-157). London: Routledge.

Benjamin, W. (1982). Das Passagen-Werk, 1983. Frankfurt am Main: Suhrkamp.

\footnotetext{
8 Jean Franco en Cruel Modernity (2013) refiere a la teoría de Enrique Dussel para plantear que uno de los paradigmas de la modernidad en América Latina fue el desarrollismo, que durante la década de los 60 y 70 se impuso como un modo de racionalización y simplificación de las relaciones económicas globales bajo la hegemonía de los Estados Unidos (Franco 2013, 5-7).
} 
Blanchot, M. (1959). El diario íntimo y el relato. In El libro que vendrá (pp. 207-212.). Trad. P. de Place. Caracas: Monte Ávila Editores, 1996.

Blanchot, M. (1969). La parole quotidienne. In L'entretien infini (pp. 355-366). Paris: Gallimard.

Cella, S. (2013). La ciudad no es una isla. Página 12. 14 de Julio. http://www.pagina12.com.ar/diario/ suplementos/libros/10-5076-2013-07-14.html. Consultado el 19 de diciembre de 2014.

Coverley, M. (2010). Psychogeography. Harpenden: Pocket Essentials.

de Certeau, M. (1980). L'invention du quotidien. Paris: Union générale d'éditions. 1990.

Debord, G. (1955). Introduction to a critique of urban geography. Trad. K. Knabb. In K. Knabb (Ed.), Situationist international anthology (pp. 24-30). Berkeley: Bureau of Public Secret. 1981.

Debord, G. (1961). Perspectives for conscious alternations in everyday life. In B. Highmore (Ed.), The everyday life reader (pp. 237-245). London: Routledge.

Felski, R. (1999-2000). The invention of everyday life. New Formations, 39, 15-31.

Fillon, J. (1954). New games! In U. Conrads (Ed.), Programs and manifestoes on 20th-century architecture (p. 155). Massachusetts: MIT Press. 1974.

Franco, J. (2013). Cruel modernity. Durham, London: Duke University Press.

Hazlitt, W. (1822). On going a journey. In The collected works of William Hazlitt (Vol. VI, pp. 181-189). London: J.M. Dent \& Co., 1903.

Highmore, B. (2002). The everyday life reader. London: Routledge.

Lalo, E. (1986). En el Burger King de la calle San Francisco. San Juan: Astrolabio.

Lalo, E. (1995). Ciudades e islas. San Juan: Yuquiyú.

Lalo, E. (2005). donde. San Juan: Tal Cual.

Lalo, E. (2012). Simone. Buenos Aires: Corregidor.

Lalo, E. (2013). Hoy es hermoso. Discurso pronunciado durante la entrega de la XVIII edición del Premio Rómulo Gallegos. Caracas: 2 de agosto. http://www.celarg.org.ve/Espanol/Imagenes/Premio\% 20Romulo\%20Gallegos/18_2013/discurso\%20lalo\%20oficial.pdf. Consultado el 15 de junio de 2014.

Lefebvre, H. (1947). Critique de la vie quotidienne. Paris: Arche. 1958.

Perec, G. (1973). Lo infraordinario. Trad. M. Cebrián. Madrid: Impedimenta. 2010.

Perec, G. (1975). Tentative d'épuisement d'un lieu parisien. Paris: Christian Bourgois. 1982.

Piglia, R., Duchesne Winter, J. R., Duno-Gottberg, y. L., (2013). Veredicto de la XVIII Edición del Premio Internacional Novela Rómulo Gallegos. Caracas: 6 de junio. http://www.celarg.org.ve/ Espanol/Imagenes/Premio\%20Romulo\%20Gallegos/18_2013/Veredicto\%20R\%C3\%B3mulo\%20Gallegos.pdf. Consultado el 15 de junio de 2014.

Plant, S. (1992). The most radical gesture. The Situationist International in a postmodern age. London, New York: Routledge.

Relph, E. (1976). Place and placelessness. London: Pion.

Sassen, S. (2007). A sociology of globalization. New York, London: W.W. Norton \& Company.

Sheringham, M. (2000). Attending to the everyday: Blanchot, Lefebvre, Certeau, Perec. French Studies, 54, 187-199.

Solnit, R. (2001). Wanderlust. A history of walking. London, New York: Verso. 2002.

Tuan, Y. (1977). Space and place. Minneapolis: University of Minnesota Press. 2003.

Winter, J. D. (2011). Noticias de un país que desaparece: 'raros' puertorriqueños de hoy. América Latina Hoy, 58, 31-50. 